\title{
Cryopreservation of Shoot Tips of Elite Cultivars of Cannabis sativa L. by Droplet Vitrification
}

\author{
Esther Uchendu ${ }^{a}$ b Hemant Lata ${ }^{a}$ Suman Chandra ${ }^{a}$ Ikhlas A. Khan ${ }^{a, c}$ \\ Mahmoud A. ElSohly a, d \\ a National Center for Natural Products Research, School of Pharmacy, University of Mississippi, University, MS, \\ USA; ${ }^{b}$ Department of Agronomy, University of Ibadan, Ibadan, Nigeria; ' Department of Pharmacognosy, School \\ of Pharmacy, University of Mississippi, University, MS, USA; 'Department of Pharmaceutics, School of Pharmacy, \\ University of Mississippi, University, MS, USA
}

\section{Keywords \\ Conservation · Cultivars · Germplasm · Liquid nitrogen · \\ Marijuana $\cdot$ Natural products}

\begin{abstract}
Cannabis sativa L. (marijuana or hemp) is recognized worldwide for its psychoactive properties as well as for fiber production. This study focused on the evaluation of 3 droplet vitrification protocols for long-term conservation of shoot tips in liquid nitrogen (LN). Shoot tips $(\sim 0.5 \mathrm{~mm})$ were excised from 3- to 4-week-old in vitro-grown shoots of 3 cultivars (MX, VI-20, and B-5: high tetrahydrocannabinol [THC], high cannabidiol [CBD], and intermediate $\mathrm{THC} \sim \mathrm{CBD}$, respectively) and pretreated on $5 \%$ dimethyl sulfoxide agar plates for $48 \mathrm{~h}$. The shoot tips were then vitrified in LN using 3 separate cryoprotectant (plant vitrification solutions [PVS] \#2, \#3, and \#4) droplets on an aluminum cryoplate. There was no significant difference between the regrowth of cryopreserved shoot tips exposed to PVS2 for 15 and $20 \mathrm{~min}$, but regrowth of all 3 cultivars significantly declined after 20 min of exposure. Exposure duration of 15 min was adapted for subsequent experiments. Regrowth of cryopreserved MX was significantly higher with PVS2 (63\%) than with PVS3 and
\end{abstract}

PVS4 ( $\leq 5 \%)$. Regrowth of cryopreserved VI-20 was highest with PVS2 (57\%) and significantly higher than with PVS3 and PVS4 ( $\leq 25 \%)$. The regrowth of cryopreserved shoot tips of B-5 was significantly different between all 3 protocols with PVS2 > PVS4 > PVS3. Both PVS2 and PVS4 produced regrowth above $55 \%$, while regrowth with PVS3 was significantly lower (31\%). These results indicate that 15-20 min of exposure to PVS2 are most suitable for cryopreservation of these varieties. This is the first report on protocol development for the cryopreservation of organized tissues of C. sativa L. for germplasm conservation.

(c) 2019 The Author(s)
Published by S. Karger AG, Basel

\section{Introduction}

Cannabis sativa is grown all over the world [1]. It has a long history of pharmacologic and therapeutic benefits due to the presence of the active cannabinoids, tetrahydrocannabinol (THC) and cannabidiol (CBD) $[2,3]$, and other constituents. Varieties with a high THC content are commonly referred to as marijuana, while those with a low THC content are known as hemp and are used for fiber [4]. In

\begin{tabular}{ll}
\hline KARGER & $\begin{array}{l}\text { (c) } 2019 \text { The Author(s) } \\
\text { Published by S. Karger AG, Basel }\end{array}$ \\
E-Mail karger@karger.com & $\begin{array}{l}\text { This article is licensed under the Creative Commons Attribution- } \\
\text { NonCommercial-NoDerivatives 4.0 International License (CC BY- }\end{array}$ \\
www.karger.com/mca & $\begin{array}{l}\text { NC-ND) (http://www.karger.com/Services/OpenAccessLicense). } \\
\text { Usage and distribution for commercial purposes as well as any dis- } \\
\text { tribution of modified material requires written permission. }\end{array}$
\end{tabular}


vitro methods for micropropagation and conservation have been reported [5-7]. The in vitro germplasm repository or genebank at the University of Mississippi, USA, was established in 2009 and has several collections of important varieties of $C$. sativa with different biochemical profiles $[6,7]$. The University of Mississippi is currently the only federally approved institution to produce bulk C. sativa for research. A cryopreservation program (storage at ultra-low temperature, usually that of liquid nitrogen $[\mathrm{LN}]$ at $-196^{\circ} \mathrm{C}$ ) has recently been established to backup or secure medicinally important varieties against loss and to ensure the future availability of desired biochemically active compounds.

Cryopreservation of plant parts began in the late 1950s and early 1960s [8], and through many decades of research, it is now possible to achieve an ice-free intracellular environment necessary to maintain plants in stable metabolic condition during long-term storage in LN. Vitrification during cryopreservation involves the phase transition of water into an amorphous, metastable glass without crystal formation. Several vitrification mixtures have been used as cryoprotectants to increase the recovery of plants after cryopreservation [9-12]. Vitrification has been applied to several types of medicinal plants with moderate to high recovery rates $[13,14]$. The droplet vitrification technique was derived from the plant vitrification solution (PVS)based vitrification technique [9] and the droplet freezing technique $[15,16]$. These techniques involve the use of a sterile aluminum cryoplate [17], an important conductor of heat or thermal energy during immersion in $\operatorname{LN}[18,19]$.

A cryopreservation technique was reported for $C$. sativa cell suspension cultures nearly 3 decades ago. Jekkel et al. [20] (1989) obtained 58\% survival after cryopreservation of the suspension cultures using a controlled cooling rate of $2{ }^{\circ} \mathrm{C} / \mathrm{min}$, transfer temperature of $-10^{\circ} \mathrm{C}$ and $10 \%$ dimethyl sulfoxide (DMSO) as the cryoprotectant. There is no cryopreservation history for shoot tips of C. sativa, which are the preferable explants for clonal micropropagation of plants. The development of a simple and reliable protocol(s) for shoot tips of C. sativa would allow widespread use of cryopreservation for banking medicinally important cultivars with unique attributes. We evaluated the effects of 3 cryoprotectants for use with the droplet vitrification protocol for effective in vitro storage of Cannabis germplasm.

\section{Materials and Methods}

Plant Material and Growth Conditions

The plant materials (shoots) used for this study were collected from the indoor-grown collections of plants produced at the $\mathrm{Na}$ tional Center for Natural Products Research (NCNPR), Coy Waller
Laboratory Complex, University of Mississippi, USA. The shoots were taken from plants of elite cultivars of C. sativa L.; MX, characterized with a high THC content, VI-20, with high CBD content; and B-5, with intermediate levels of THC and CBD content. These indoor-grown plants were raised under climatic control conditions (temperature: $75 \pm 3^{\circ} \mathrm{F}$, and relative humidity: $\sim 60 \%$ ).

\section{Sterilization}

The shoots were pre-rinsed for $2 \mathrm{~min}$ in running tap water, followed by surface disinfection in $15 \%$ commercial bleach (Clorox, regular bleach $[5.25 \% v / v]$ ) with $0.1 \%$ Tween 20 for $18 \mathrm{~min}$ and then rinsed thoroughly with sterile distilled water $(500 \mathrm{~mL})$ for 3 $\min$. The shoots were further treated with $0.2 \%$ mercuric chloride for $3 \mathrm{~min}$. Shoots were rinsed 3 times with sterile distilled water before planting in growth medium.

\section{Growth Medium Composition}

After the surface sterilization of shoots, they were transferred to growth medium containing Murashige and Skoog (MS) mineral salts [21] formulated as a commercial powder (Caisson Laboratories Inc.) and MS vitamins mixture, with $0.12 \mathrm{mgL}^{-1}$ metatopolin (Caisson Laboratories Inc.), $8 \mathrm{gL}^{-1}$ agar powder (Caisson Laboratories Inc.), $30 \mathrm{gL}^{-1}$ anhydrous sucrose (Caisson Laboratories Inc.), and $1 \mathrm{gL}^{-1}$ activated charcoal. The $\mathrm{pH}$ of the medium was adjusted to 5.7 before the addition of the agar and activated charcoal. The medium was sterilized by autoclaving at $121^{\circ} \mathrm{C}$, for $15 \mathrm{~min}$, and dispensed after autoclaving into sterile Magenta GA7 boxes.

\section{Culture Conditions}

All shoot cultures were transferred to a culture room with controlled environmental conditions of $25 \pm 2{ }^{\circ} \mathrm{C}$ at a 16 -h photoperiod. The shoots were grown under photon flux of $52 \mu \mathrm{mol} / \mathrm{m}^{2} / \mathrm{s}$ (LI-250A, LI-COR ${ }^{\circledR}$ Biosciences, USA) provided by cool white fluorescent bulbs. The shoots were subcultured on fresh growth medium every 3-4 weeks.

\section{Cryopreservation Procedure}

Pretreatment Procedure

Shoot tips $(\sim 0.5 \mathrm{~mm})$ consisting of a meristematic dome with 1 or 2 attached leaf primordia were excised from 3-week-old shoot cultures and held for $48 \mathrm{~h}$ on a pretreatment medium consisting of MS basal medium with $0.3 \mathrm{M}$ sucrose, $8 \mathrm{gL}^{-1}$ agar, and 5\% DMSO (Sigma-Aldrich Co.) at $\mathrm{pH} 5.7$ prior to all experimentation. The DMSO was filter sterilized using membrane filters $(0.45 \mu \mathrm{m}, 150$ $\mathrm{mL}$ analytical filter unit) and added to a cooled pretreatment medium after autoclaving. About $25 \mathrm{~mL}$ medium was dispensed into each Petri dish. The shoot tip cultures were placed in the grow room under the controlled conditions described above. After the pretreatment, shoot tips of each variety were cryopreserved, with shoot tips treated but not exposed to LN used as the controls.

\section{Composition of Cryoprotectants}

The cryoprotectants (vitrification solutions) consisted of PVS2 (30\% glycerol, 15\% ethylene glycol, 15\% DMSO in liquid MS medium with $0.4 \mathrm{M}$ sucrose $[w / v], \mathrm{pH} 5.8$ ) [9], PVS3 (40\% sucrose, $40 \%$ glycerol in liquid MS medium $[w / v], \mathrm{pH} 5.8$ ) [11], and PVS4 (0.6 M sucrose, $3.8 \mathrm{M}$ glycerol, and $20 \%$ ethylene glycol in liquid MS $[w / v]$, pH 5.8) [22]. 

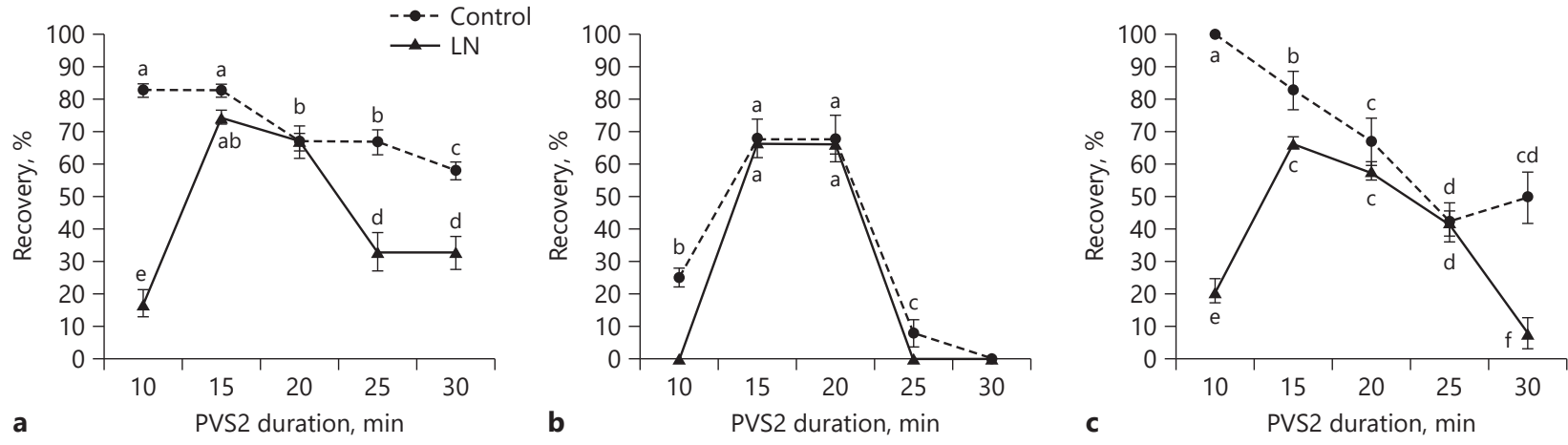

Fig. 1. Regrowth rates of shoot tips of Cannabis sativa cultivars MX (a), VI-20 (b), and B-5 (c) exposed to plant vitrification solution \#2 (PVS2) for $10-30 \mathrm{~min}$ at $25 \pm 2{ }^{\circ} \mathrm{C}$ prior to cryopreservation in liquid nitrogen (LN). The controls had all treatments except LN. Bars with the same letter are nonsignificant at $p \leq 0.05$
Effect of Cryoprotectants

The optimal exposure durations for PVS2, PVS3, and PVS4 were determined. Shoot tips of each variety (12 per treatment) were pretreated as described above and transferred to $1 \mathrm{~mL}$ loading solution (LS) (2 M glycerol in $0.4 \mathrm{M}$ sucrose MS medium $[v / v], \mathrm{pH}$ 5.8) [22] in $1.2 \mathrm{~mL}$ cryovials for $20 \mathrm{~min}$ at $25 \pm 1^{\circ} \mathrm{C}$. The LS was removed and the shoot tips were individually transferred to wells on a sterile aluminum cryoplate $(7 \times 37 \times 0.5 \mathrm{~mm}$, depth of well is $0.75 \mathrm{~mm}$ ) with 3 - to 5 - $\mu \mathrm{L}$ droplets of cryoprotectant solutions per well. Each aluminum cryoplate had 12 wells with 1 shoot tip per well. Cryoprotectants were applied at $25 \pm 1{ }^{\circ} \mathrm{C}$ for $10,15,20,25$, and $30 \mathrm{~min}$. Following each of these exposure durations, 12 shoot tips per treatment per replicate were rinsed in liquid MS medium containing $1.2 \mathrm{M}$ sucrose as described below and then cultured in the growth medium (control). Also, 12 shoot tips per treatment per replicate were plunged rapidly in LN for 10-15 min, followed by rapid rewarming in a $45^{\circ} \mathrm{C}$ water bath for $1 \mathrm{~min}$ before the samples were rinsed in a solution of liquid MS with $1.2 \mathrm{M}$ sucrose and then planted on above growth medium in 24-cell culture plates. This experiment was done with 3 replicates for each variety.

Testing of Vitrification Procedures on C. sativa

The droplet vitrification procedure of Panis et al. [23] was followed. The successive steps for the cryopreservation of $C$. sativa shoot tips of each variety were as follows: (1) pretreatment of shoot tips in 5\% DMSO plate for $48 \mathrm{~h}$. (2) LS was added to shoot tips as described above for $20 \mathrm{~min}$. (3) The shoot tips (1 per well) were transferred into sterile aluminum cryoplates with droplets of each cryoprotectant (\#2,\#3, and \#4). Each treatment had 20 shoot tips per treatment per replicate and was held in the cryoprotectant for 15 min. (4) Aluminum cryoplates with shoot tips were transferred to a cryovial, closed, and rapidly plunged in LN for 10-15 min. (5) Cryopreserved shoot tips were rapidly rewarmed in $45^{\circ} \mathrm{C}$ water for $1 \mathrm{~min}$. (6) Shoot tips were rinsed by serial dilution in liquid MS medium

Cryopreservation of Cannabis sativa L. by Droplet Vitrification containing 1.2 M sucrose for 3-5 min. (7) Cryopreserved shoot tips were planted in the standard growth medium described above. The controls (12 shoot tips) had the same treatments but were not plunged into LN. Each cultivar and treatment was tested separately.

\section{Data Collection}

Data on the regrowth of shoot tips were recorded 8 weeks after rewarming. Regrowth was recorded for each originally cryopreserved shoot tip that remained green, enlarged, and resumed growth with production of leaves and shoots. All data are presented as percentage of total number of original shoot tips that recovered from cryopreservation \pm SE of means. Data were analyzed using ANOVA (SAS version 9.2 for Windows; SAS Institute Inc., Cary, NC, USA), followed by means separation using Duncan's multiple range test. Differences in means were considered significant at $p \leq 0.05$.

\section{Results}

Effect of Cryoprotectants Duration on the Regrowth of Cryopreserved Shoot Tips

There was no significant difference between the regrowth of cryopreserved shoot tips exposed to PVS2 for 15 and 20 min (Fig. 1). However, there was generally a significant decline in regrowth beyond $20 \mathrm{~min}$ in PVS2. Variety MX (Fig. 1a) had more regrowth at extreme exposure $(30 \mathrm{~min})$ than other cultivars. Exposure durations of 10,25 , and 30 min resulted in $0 \%$ regrowth following cryopreservation of variety VI-20 (Fig. 1b). Variety B-5 (Fig. 1c) appeared more tolerant to PVS2 with better re- 


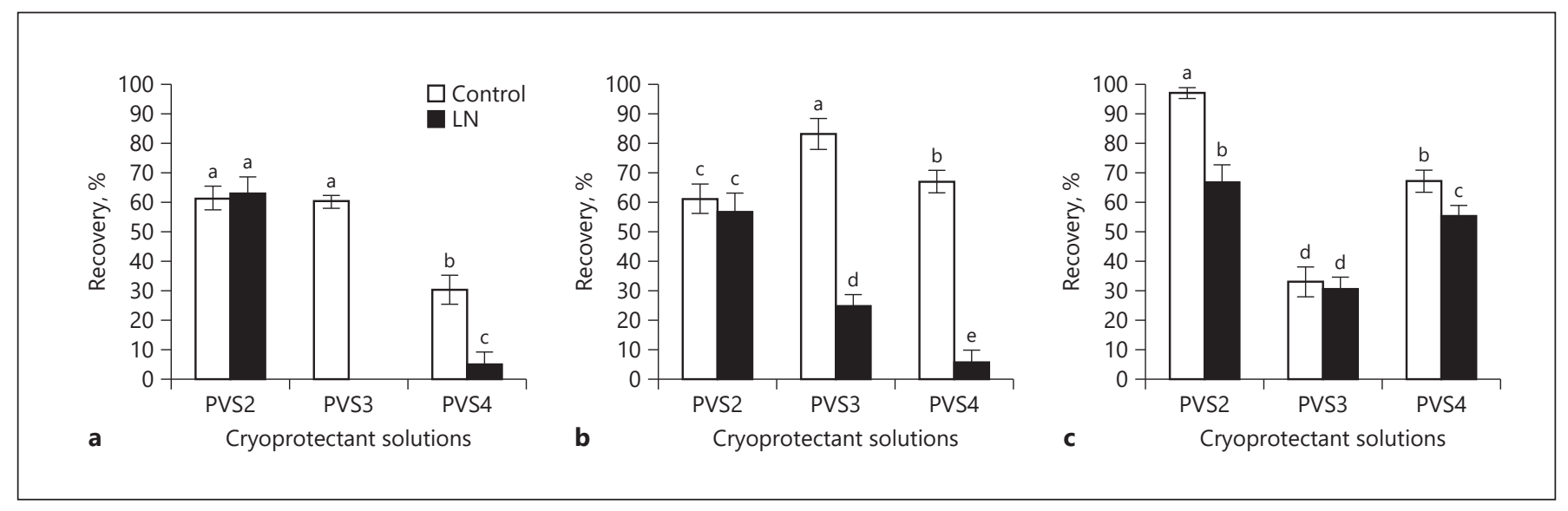

Fig. 2. Regrowth of shoot tips of Cannabis sativa cultivars MX (a), VI-20 (b), and B-5 (c) exposed to plant vitrification solutions (PVS) \#2, \#3, and \#4 for $15 \mathrm{~min}$ at $25 \pm 1{ }^{\circ} \mathrm{C}$ prior to droplet vitri- fication cryopreservation in liquid nitrogen (LN). The controls had all treatments except exposure to LN. Bars with the same letter are nonsignificant at $p \leq 0.05$.
Fig. 3. Regrowth after 6 weeks of rewarming of cryopreserved shoot tips $(\sim 0.5 \mathrm{~mm})$ of Cannabis sativa cultivar B-5. Shoot tips were cryopreserved by droplet vitrification using plant vitrification solutions (PVS) \#2, \#3, and \#4. a Survival indicated cryopreserved shoot tips that remained green and/ or enlarged but did not develop. b Regrowth of cryopreserved shoot tips with differentiated leaves and shoots.

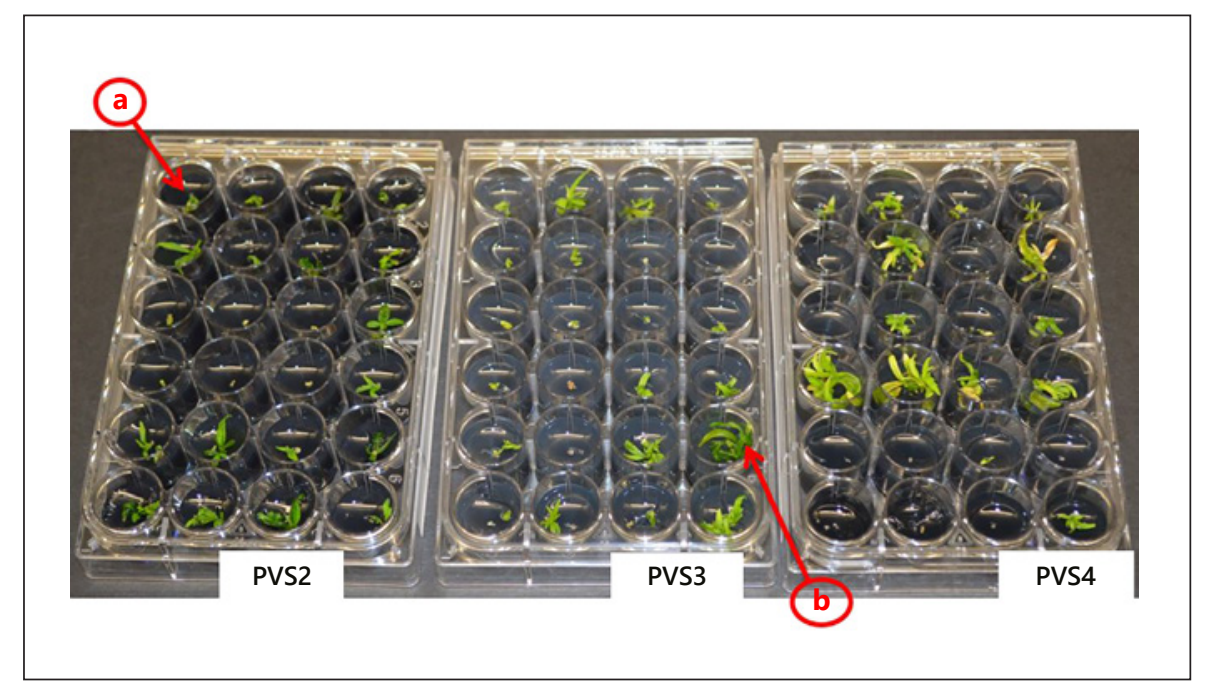

growth rates after cryopreservation than variety VI-20. The cultivars had comparatively poor regrowth with PVS3 and PVS4 (data not shown).

\section{Effect of Cryoprotectants on the Droplet Vitrification of C. sativa Cultivars}

Regrowth percentage of MX shoot tips following $\mathrm{LN}$ was 63\% with PVS2 treatment (Fig. 2a), while it was less than $10 \%$ with PVS4, and there was no regrowth from PVS3, although the control was high. Regrowth of control shoot tips without LN was significantly better with PVS2 (61\%) and PVS3 than with PVS4 (30\%); however, there was no significant difference between PVS2 and PVS3 (Fig. 2a).
Regrowth of VI-20 shoot tips following cryopreservation was highest with PVS2 (57\%). This was significantly higher than with PVS3 (25\%) and PVS4 (6\%). The nonfrozen shoot tips had regrowth of $61-80 \%$ for all 3 cultivars (Fig. 2b).

The regrowth of cryopreserved B-5 shoot tips was significantly different with PVS2 > PVS4 > PVS3. Both PVS2 and PVS4 had regrowth above 50\%, but with PVS3 regrowth was low (31\%) (Fig. 2c). The regrowth of noncryopreserved B-5 shoot tips treated with PVS2 was significantly better than with other treatments (Fig. 2c).

There was no intermediary callus formation during recovery from any of the cultivars, but a few low-quality 
shoot tips were observed that remained green and/or enlarged ( $\geq 3 \mathrm{~mm}$ in length) but did not undergo differentiation (Fig. 3a).

\section{Discussion}

One of the important keys to a successful cryopreservation by vitrification is to optimize the dehydration process in cryoprotectant solution in order to prevent injury by chemical toxicity or osmotic shock resulting in increased regrowth. We examined the regrowth responses of cryopreserved shoot tips of all 3 Cannabis varieties over a range of exposure durations to PVS2, a cryoprotectant generally known to produce time-dependent regrowth following cryopreservation $[19,24]$, and found that the cryopreserved shoot tips of these varieties produced significantly higher regrowth at 15 and $20 \mathrm{~min}$ compared to other durations (Fig. 1). Many cryopreserved medicinal plants have optimal regrowth when exposed for $20 \mathrm{~min}$ to PVS2 [14, 25]. However, 10-25 min exposure duration to PVS2 at $25^{\circ} \mathrm{C}$ was shown to be optimal for several herbaceous plant species $[24,26]$. It is clear from these studies that the acquisition of tolerance to PVS2 is necessary for survival after cryopreservation and also that the exposure duration may be genotype dependent. Our data detail the specific regrowth responses of both cryopreserved and noncryopreserved shoot tips of C. sativa exposed to PVS2 and the role of cryoprotection in Cannabis recovery from LN. Exposure of shoot tips of these cultivars for a longer time ( $>30 \mathrm{~min}$ ) to PVS3 and PVS4 may be necessary to achieve sufficient cryoprotection and improved regrowth. Kim et al. [27] (2009) evaluated 90-150 min exposure of garlic explants to PVS3 and found that 150 min was optimal for cryopreservation of garlic by droplet vitrification.

The MX is a unique cultivar with high THC content [2]. This implies that the cultivar is of great importance for drug production. The ability to successfully cryopreserve this cultivar with over $60 \%$ regrowth, using the PVS2 protocol (Fig. 2a), provides long-term security for this and other important varieties processed for in vitro conservation. Overall, the PVS2 droplet vitrification customized for Cannabis spp. produced a moderate regrowth of $57-67 \%$ of cryopreserved shoot tips. This result indicates that this technique could be applied on all 3 varieties targeted for long-term conservation. This project is part of an ongoing effort to cryopreserve all the varieties of $C$. sativa held at the NCNPR, University of Mississippi. The droplet vitrification technique using PVS2 has been applied to many medicinal plant types, including Byrsonima

Cryopreservation of Cannabis sativa L. by

Droplet Vitrification intermedia [28] and Atractylodes macrocephala [29]. The significance of each critical step of this vitrification procedure has been reviewed $[19,30]$.

An important observation was made during the recovery of cryopreserved shoot tips. The majority of the cryopreserved shoot tips of these varieties had as much as $83 \%$ survival (data not shown), but in many cases this did not translate or equate to regrowth (Fig. 3b). Similar observations were made during the cryopreservation of Lilium lancifolium by droplet vitrification [31]. The authors recorded $84 \%$ survival, while the regrowth was $68 \%$. Kim et al. [12] (2006) reported $60.7 \%$ survival and a regrowth of $50.2 \%$ for garlic shoot tips and also $69.1 \%$ survival and a regrowth of $30.8 \%$ for Chrysanthemum shoot tips following cryopreservation by the PVS2 droplet technique. Leunufna and Keller [32] (2005) obtained higher survival (67-70\%) compared to regrowth (30-50\%) of cryopreserved Dioscorea spp. following a modified PVS2 droplet vitrification technique. A low regrowth compared to survival rate is not peculiar to droplet vitrification alone. Preetha [25] (2013) reported $50-60 \%$ survival and $30-40 \%$ regrowth after cryopreservation of the medicinal plant Kaempferia galanga L. by the regular PVS2 vitrification technique. The causes of this phenomenon (low rates of differentiation of cells and tissues) during vitrification or droplet vitrification methods are unclear, however; the major factors affecting the growth and development of cells or tissues after cryopreservation include the physiological status of the donor plant [33] and the explant to be cryopreserved [34], size and position of shoot tips [35], and origin of material [36]. Due to the regulatory nature of $C$. sativa, these factors and mechanisms have not been fully explored. Research on different aspects of cryopreservation is ongoing in our laboratory and will be communicated in the future. This study was initiated in the hope to achieve protocol development for cryopreservation of Cannabis tissues. The results are encouraging.

\section{Acknowledgements}

This project was supported in part by the National Institute of Drug Abuse (NIDA). Contract \# N01DA-15-7793.

\section{Statement of Ethics}

The authors have no ethical conflicts to disclose.

\section{Disclosure Statement}

The authors have declared no conflict of interest.
Med Cannabis Cannabinoids 2019;2:29-34 DOI: $10.1159 / 000496869$ 


\section{References}

1 Small E, Marcus D. Hemp: A new crop with new uses for North America. In: Janick J, Whipkey A, editors. Trends in new crops and new uses. Alexandria (VA): ASHS Press; 2002. pp. 284-326.

2 Chandra S, Lata H, Mehmedic Z, Khan IA, ElSohly MA. Assessment of cannabinoids content in micropropagated plants of Cannabis sativa and their comparison with conventionally propagated plants and mother plant during developmental stages of growth. Planta Med. 2010 May;76(7):743-50.

3 Zuardi AW. History of cannabis as a medicine: a review. Br J Psychiatry. 2006 Jun;28(2): 153-7.

4 Slade D, Mehmedic Z, Chandra S, ElSohly M. Is cannabis becoming more potent? In: Castle D, Murray RM, D'Souza DC, editors. Marijuana and Madness. 2nd ed. Cambridge: Cambridge University Press; 2012. pp. 35-54.

5 Mandolino G, Ranalli P. Advances in biotechnological approaches for hemp breeding and industry. In: Ranalli P, editor. Advances in hemp research. New York: Haworth Press; 1999. pp. 185-208.

6 Lata H, Chandra S, Khan I, ElSohly MA. Thidiazuron-induced high-frequency direct shoot organogenesis of Cannabis sativa L. In Vitro Cell Dev Biol Plant. 2009;45(1):12-9.

7 Lata H, Chandra S, Mehmedic Z, Khan IA, ElSohly MA. In vitro germplasm conservation of high $\Delta 9$-tetrahydrocannabinol yielding elite clones of Cannabis sativa L. under slow growth conditions. Acta Physiol Plant. 2012;34(2):743-50.

8 Sakai A. Survival of the twig of woody plants at $-196^{\circ} \mathrm{C}$. Nature. $1960 ; 185(4710): 393-4$.

9 Sakai A, Kobayashi S, Oiyama I. Cryopreservation of nucellar cells of navel orange (Citrus sinensis Osb. var. brasiliensis Tanaka) by vitrification. Plant Cell Rep. 1990 Jun;9(1): 30-3.

10 Sakai A, Kobayashi S, Oiyama I. Survival by vitrification of nucellar cells of navel orange (Citrus sinensis var. brasiliensis Tanaka) cooled to $-196^{\circ}$ C. J Plant Physiol. 1991; 137(4):465-70.

11 Nishizawa S, Sakai A, Amano Y, Matsuzawa T. Cryopreservation of asparagus (Asparagus officinalis L.) embryogenic suspension cells and subsequent plant regeneration by vitrification. Plant Sci. 1993;91(1):67-73.

12 Kim HH, Lee JK, Yoon JW, Ji JJ, Nam SS, Hwang HS, et al. Cryopreservation of garlic bulbil primordia by the droplet-vitrification procedure. Cryo Letters. 2006 May-Jun;27(3): 143-53.
13 Uchendu EE, Brown DW, Saxena PK. Cryopreservation of shoot tips and cotyledons of the North American ginseng (Panax quinquefolius L.). Cryo Letters. 2011 Nov-Dec;32(6): $463-72$.

14 Uchendu EE, Shukla MR, Reed BM, Saxena PK. An efficient method for cryopreservation of St John's wort and tobacco: role of melatonin. Acta Hortic. 2014;(1039):23341.

15 Kartha KK, Leung NL, Mroginski LA. In vitro growth responses and plant regeneration from cryopreserved meristems of cassava (Manihot esculenta Crantz). Z Pflanzenphysiol. 1982;107(2):133-40.

16 Schäfer-Menuhr A, Schumacher HM, MixWagner G. Cryopreservation of potato cultivars: design of a method for routine application in genebanks. Acta Hortic. 1997;(447): $477-82$.

17 Matsumoto T. Cryopreservation of plant genetic resources: conventional and new methods. Rev Agric Sci. 2017;5(0):13-20.

18 Panis B, Piette B, Swennen R. Droplet vitrification of apical meristems: a cryopreservation protocol applicable to all Musaceae. Plant Sci. 2005; 168(1):45-55.

19 Sakai A, Engelmann F. Vitrification, encapsulation-vitrification and droplet-vitrification: a review. Cryo Letters. 2007 May-Jun;28(3): 151-72.

20 Jekkel Z, Heszky LE, Ali AH. Effect of different cryoprotectants and transfer temperatures on the survival rate of hemp (Cannabis sativa L.) cell suspension in deep freezing. Acta Biol Hung. 1989;40(1-2):127-36.

21 Murashige T, Skoog F. A revised medium for rapid growth and bio assays with tobacco tissue cultures. Physiol Plant. 1962;15(3):47397.

22 Sakai A. Development of cryopreservation techniques. In: Engelmann F, Takagi H, editors. Cryopreservation of Tropical Plant Germplasm - Current Research Progress and Application. Rome: JIRCAS, Tsukuba and IPGRI; 2000. pp. 1-7.

23 Panis B, Piette B, André E, van den Houwe I, Swennen R. Droplet vitrification: the first generic cryopreservation protocol for organized plant tissues? Acta Hortic. 2011;(908):15762.

24 Matsumoto T, Sakai A, Yamada K. Cryopreservation of in vitro-grown apical meristems of wasabi (Wasabia japonica) by vitrification and subsequent high plant regeneration. Plant Cell Rep. 1994 May;13(8): 442-6.
25 Preetha TS. Shoot tip cryopreservation by vitrification in Kaempferia galanga L. An endangered, overexploited medicinal plant in Tropical Asia. IOSR J Pharm Biol Sci. 2013;8(3):19-23.

26 Takagi H, Tien Thinh N, Islam OM, Senboku T, Sakai A. Cryopreservation of in vitrogrown shoot tips of taro (Colocasia esculenta (L.) Schott) by vitrification. 1. Investigation of basic conditions of the vitrification procedure. Plant Cell Rep. 1997;16(9):594-9.

27 Kim HH, Lee YG, Shin DJ, Ko HC, Gwag JG, Cho EG, et al. Development of alternative plant vitrification solutions in droplet-vitrification procedures. Cryo Letters. 2009 SepOct;30(5):320-34.

28 Silva LC, Paiva R, Swennen R, Andre E, Panis B. Shoot-tip cryopreservation by droplet vitrification of Byrsonima intermedia A. Juss.: a woody tropical and medicinal plant species from Brazilian cerrado. Cryo Letters. 2013 Jul-Aug;34(4):338-48.

29 Zhang JM, Huang B, Lu XX, Volk GM, Xin X, Yin GK, et al. Cryopreservation of in vitrogrown shoot tips of Chinese medicinal plant Atractylodes macrocephala Koidz. using a droplet-vitrification method. Cryo Letters. 2015 May-Jun;36(3):195-204.

30 Sakai A, Hirai D, Niino T. Development of PVS-based vitrification and encapsulationvitrification protocols. In: Reed BM, editor. Plant Cryopreservation: A Practical Guide. New York: Springer; 2008. pp. 33-57.

31 Chen XL, Li JH, Xin X, Zhang ZE, Xin PP, Lu $\mathrm{XX}$. Cryopreservation of in vitro-grown apical meristems of Lilium by droplet-vitrification. S Afr J Bot. 2011;77(2):397-403.

32 Leunufna S, Keller ER. Cryopreservation of yams using vitrification modified by including droplet method: effects of cold acclimation and sucrose. Cryo Letters. 2005 Mar-Apr; 26(2):93-102.

33 Reed BM, editor. Plant Cryopreservation: A Practical Guide. New York: Springer; 2008.

34 Dereuddre J, Fabre J, Bassaglia C. Resistance to freezing in liquid nitrogen of carnation (Dianthus caryophyllus L. var Eolo) apical and axillary shoot tips excised from different aged in vitro plantlets. Plant Cell Rep. 1988 May;7(3):170-3.

35 Azimi-Tabrizi M, O'Brien C, Ashmore S, Drew RA. Cryopreservation of papaya germplasm. Acta Hortic. 2005;(692):43-50.

36 Baek HJ, Kim HH, Cho EG, Chae YA, Engelmann F. Importance of explant size and origin and of preconditioning treatments for cryopreservation of garlic shoot apices by vitrification. Cryo Letters. 2003 Nov-Dec;24(6):381-8. 